\title{
The role of neuregulin-1 in the response to nerve injury
}

\section{Florence R Fricker ${ }^{\dagger 1}$ \& David LH Bennett}

'Wolfson CARD, King's College London, Guy's Campus, London SE1 1UL, UK

†Author for correspondence: Tel.: +44 2078486179 m Fax: +44 2078486165 = florence.fricker@kcl.ac.uk

Axons and Schwann cells exist in a highly interdependent relationship: damage to one cell type invariably leads to pathophysiological changes in the other. Greater understanding of communication between these cell types will not only give insight into peripheral nerve development, but also the reaction to and recovery from peripheral nerve injury. The type III isoform of neuregulin-1 (NRG1) has emerged as a key signaling factor that is expressed on axons and, through binding to erbB2/3 receptors on Schwann cells, regulates multiple phases of their development. In adulthood, NRG1 is dispensable for the maintenance of the myelin sheath; however, this factor is required for both axon regeneration and remyelination following nerve injury. The outcome of NRG1 signaling depends on interactions with other pathways within Schwann cells such as Notch, integrin and CAMP signaling. In certain circumstances, this signaling pathway may be maladaptive; for instance, direct binding of Mycobacterium leprae onto erbB2 receptors produces excessive activation and can actually promote demyelination. Attempts to modulate this pathway in order to promote nerve repair will therefore need to give consideration to the exact isoform used, as well as how it is processed and the context in which it is presented to the Schwann cell.

Peripheral nerve injury causes significant morbidity and reduced quality of life as a consequence of weakness, sensory loss and neuropathic pain. In total, $6 \%$ of the elderly population suffer from peripheral neuropathy [1], which can be caused by metabolic diseases such as diabetes, inherited genetic disorders such as Charcot-Marie-Tooth disease, infectious and inflammatory disorders including Guillan-Barré syndrome and traumatic injury to the nerve (which alone effects up to 300,000 people in Europe per year [1,2]). In contrast to the CNS, peripheral nerves have a significant regenerative capacity [3]; however, in patients, regeneration and the clinical outcome are often poor. Axons regenerate at a rate of approximately $1 \mathrm{~mm}$ per day, depending on the site of the lesion. There may be a delay of months if not years before the target zone is reinnervated, and by this time, the target organ and supporting cells may have irreversibly changed, becoming much less receptive to re-innervation. The current approaches to peripheral nerve repair following nerve transection are either to surgically suture the nerve or to bridge the gap between the two cut ends. Bridging the gap can be achieved with either a nerve auto- or allo-graft or a conduit (reviewed in [4]). As yet, there are no pharmacological therapies that increase the rate of axon regeneration, improve target specificity or prevent atrophy of target organs. Following nerve injury, coordinated signaling between axons, Schwann cells (SCs) and macrophages are required for effective nerve repair. For instance, axons traversing a nerve gap have been shown to require close association with migrating SCs [5]. Greater understanding of such signaling events may give new opportunities to enhance nerve repair. It has recently been demonstrated that neuregulin-1 (NRG1) is an essential axoglial signal required for peripheral nerve development, and there is now growing evidence that NRG1 is required for effective nerve repair.

\section{NRGs \& the PNS \\ NRGs}

Neuregulins are a family of growth factors encoded by four genes (NRG1-4) all of which act through erbB tyrosine kinase receptors and contain EGF - like domains, which are essential for their activity [6-10]. NRG1 is by far the most extensively characterized of the NRG1 family and is the focus of this article. Historically different isoforms were described simultaneously and named depending on their observed functions: acetylcholine receptor-inducing activity [11], glial growth factor [8,12], heregulin [13] and neu differentiation factor (Neu). These names are still occasionally used in the literature [14]. Marchionni and coworkers were the first group to recognize that all these factors were products of the same gene and proposed this gene was named $N R G[8]$. The human $N R G 1$ gene is extensive, spanning approximately $1.4 \mathrm{mb}$ [15]. At least 31 isoforms of human NRG1 have been described, generated

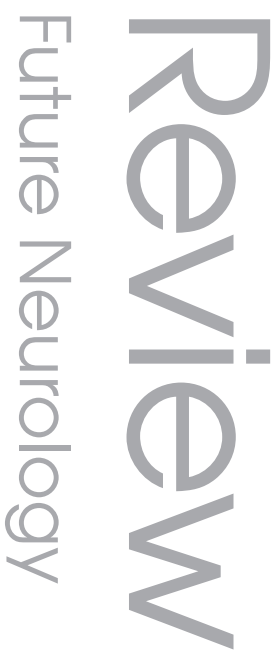

\section{Keywords}

- neuregulin-1 $=$ neuropathy

- peripheral nerve injury

- regeneration - remyelination

- repair $₫$ Schwann cell

\section{future ferot $_{\text {medicine }}$ fsg}


by way of alternative splicing and use of differential transcription start sites [16]. Isoforms differ in their $\mathrm{N}$-terminal motifs, and based on these differences, NRG1 isoforms are categorized into six types, type I-VI. Functionally, the most widely studied isoform subtypes have been types I-III, while little is yet known about the more recently described types IV-VI [16-18].

Type I, II, IV and V isoforms contain a single transmembrane domain and immunoglobulin (Ig)-like domains in their $\mathrm{N}$-terminus, hence they are sometimes referred to as the Ig-NRGs. Type II isoforms also have a kringle-like domain in their N-terminus (Figure 1). The function of the Ig-like domains is thought to lie in their high affinity for binding to the heparin-sulfate proteoglycans present on the cell surface and in the extracellular matrix. By interacting with heparinsulfate proteoglycans, Ig-NRGs are thought to be concentrated in the vicinity of erbB receptors and therefore enhance signaling $[19,20]$. Proteolytic cleavage of NRG1 types I, II and IV from the membrane releases a mature NRG1 as a diffusible signal that activates erbB receptors on nearby cells, thus signaling in a paracrine fashion [15]. Type III isoforms contain two transmembrane domains and a cysteine-rich domain in the $\mathrm{N}$-terminus. NRG1 type III is not secreted following cleavage; its second transmembrane domain tethers it to the membrane. A second cleavage event releasing NRG1 type III would be theoretically possible; however, biochemical analysis by accelerating ectodomain shedding in culture did not result in an increase in released soluble NRG1 type III, suggesting the mature form remains anchored to the membrane (FIGURE 1) [21]. Consequently NRG1 type III can only activate erbB receptors on cells it is directly in contact with, and therefore NRG1 type III signals in a juxtacrine fashion. Juxtacrine NRG1 signaling has been shown to be crucial for the development of the PNS, a topic that will be discussed in more detail later [15].

Proteolytic cleavage of NRGs is known to be catalyzed by the $\beta$-secretase $\beta$-site of amyloid precursor protein-cleaving enzyme (BACE-1 or memapsin 2) and the $\alpha$-secretases belonging to the 'a disintegrin and metalloprotease' (ADAM) family; ADAM10, ADAM19 (meltin $\beta$ ) and TNF- $\alpha$-converting enzyme (TACE or ADAM17). Cleavage of NRG1 by BACE-1 and TACE is positively regulated by nardilysin (NRDc) [22-28]. BACE-1 and TACE are known to cleave NRG1 type III at distinct cleavage sites and, interestingly, BACE-1 positively regulates myelination and TACE negatively regulates myelination [25-27,29].
Neuregulins signal through erbB tyrosine kinase receptors and erbB receptors function through dimers of erbB2, erbB3 and erbB 4 subunits, with erbB4 being the only subunit capable of forming a functional homodimer as erbB3 is catalytically inactive and erbB2 does not possess an extracellular binding domain. The expression and composition of erbB receptors is spatially and temporally regulated, NRG1 type I, II and III are all expressed in the PNS and the predominant erbB receptors expressed are erbB2 and erbB3; consequently, the erbB2/3 heterodimer is the main PNS NRG1 receptor expressed in SCs [30-32].

On activation by a NRG1 ligand, erbB receptors undergo a conformational change, dimerize and tyrosine residues present in the intracellular domains are phosphorylated. These residues then act as docking sites for adaptor proteins and enzymes, eliciting downstream signaling cascades that eventually lead to changes in transcription [31]. The composition of the erbB receptor and the bound NRG1 isoform affects the residues that are phosphorylated and consequently the adaptor proteins that can bind to them, thus determining the pathway that is activated. Signaling pathways known to be activated by NRG1 signaling in the PNS will be discussed later.

\section{The role of NRG1 in peripheral nerve development}

Development of the PNS requires the coordinated signaling between axons and glial cells in order to match the number of SCs to axons, both for the process of myelination of large-diameter axons by myelinating SCs and for enclosure of small-diameter axons in SC pockets of nonmyelinating SCs. NRG1 is known to be an important signaling factor at virtually all stages of SC development, from migratory neural crest cells to the formation of mature SCs [33,34].

Neuregulin-1 signaling is critical in SC precursor (SCP) generation. Transgenic animals lacking NRG1 isoforms or erbB2/3 have a complete absence of SCPs in the developing peripheral nerves [35-41]. By contrast, in Ig mutants lacking NRG1 type I and II, SCPs develop normally, implicating NRG1 type III as the major isoform required for development of SCPs [32]. From these knockout experiments and in vitro data, it seems likely that NRG1 signaling is necessary for migration of SCPs beyond the dorsal root ganglia (DRG) into the peripheral nerves and their survival before they mature into immature SCs $[36,38,41-43]$. SCP survival has been found to be 


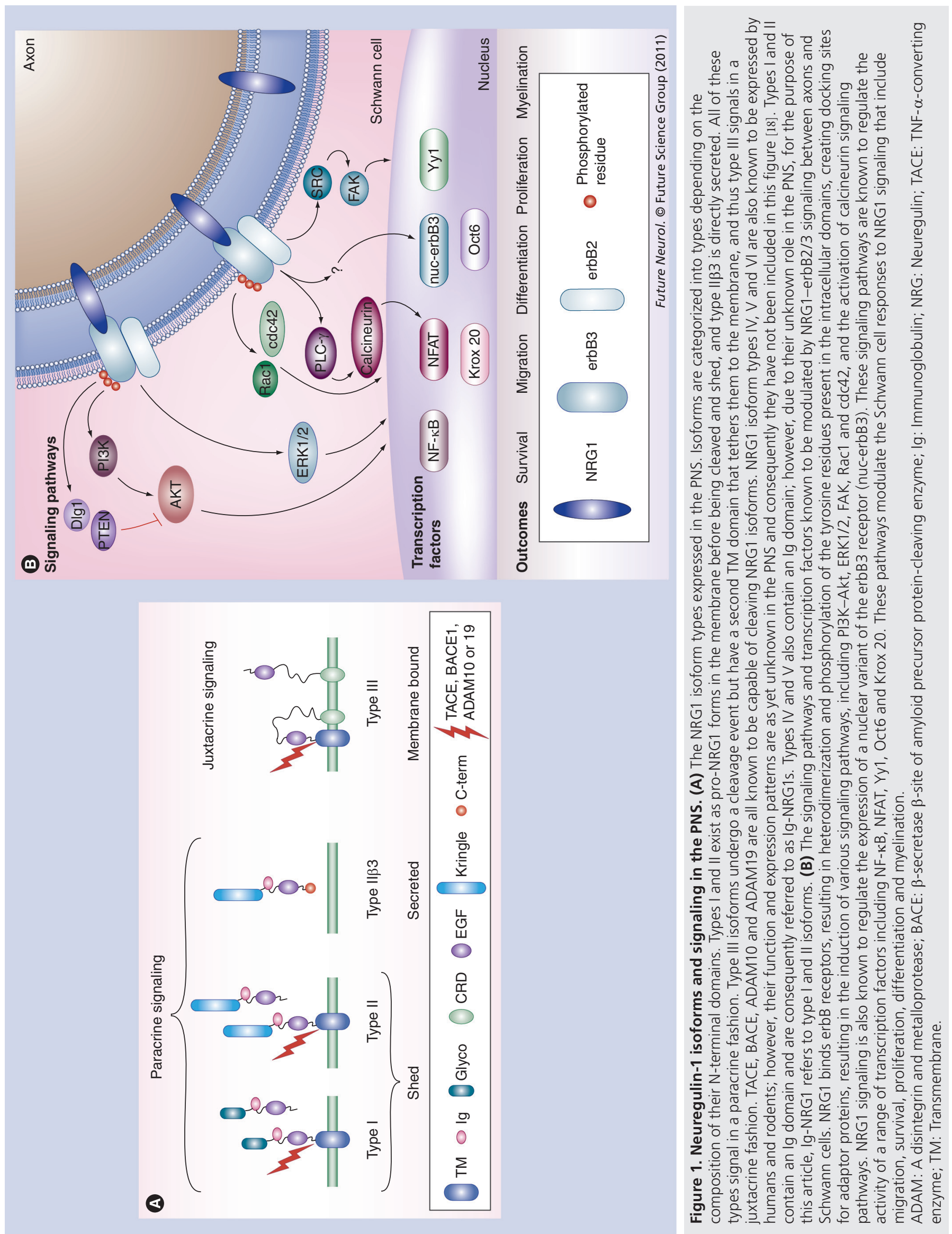




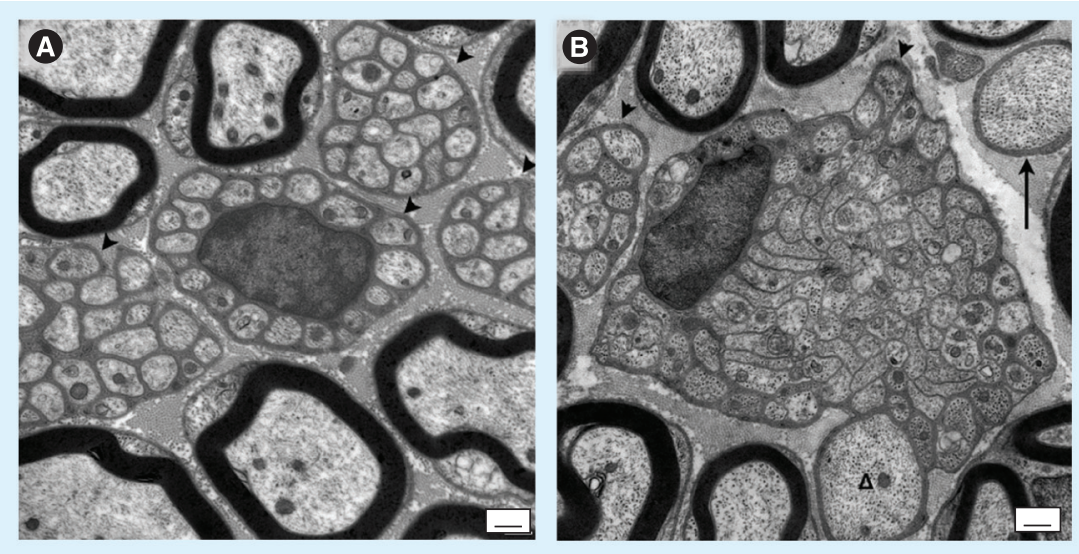

Figure 2. Neuregulin-1 is required for axoglial signaling between axons and both myelinating Schwann cells and nonmyelinating Schwann cells. Electron micrographs of transverse sections of sural nerve from control mice (A) and mice in which neuregulin-1 (NRG1) is ablated in sensory axons during development using a Nav1.8 promoter-driven Cre recombinase (B). In the absence of NRG1, Remak bundles (arrow heads) are abnormally large, axons are not all separated by Schwann cell processes forming polyaxonal pockets and large diameter axons are present within the Remak bundle $\Delta$ (B). Such abnormal Remak bundles illustrate the importance of NRG1 in signaling between nonmyelinating Schwann cells and axons. There is also a small proportion of thinly myelinated axons (that express Nav1.8-Cre) in which NRG1 is ablated; the arrow identifies an axon that would usually be myelinated but is completely amyelinated. However, this axon is invested in Schwann cell cytoplasm and a basement membrane surrounds the fiber (B). Scale bars: $500 \mathrm{~nm}$.

Reproduced with permission from [56].

mediated by axonally derived soluble NRG1 [44], which also acts as a positive regulator in the generation of immature SCs from SCPs. There is a crucial bidirectional axoglial relationship during development, which is mediated via growth factor signaling. For instance, SCPs release brainderived neurotrophic factor, which binds to trkB receptors on axons, leading to enhanced release of soluble NRG1 and so promoting survival of SCPs and, conversely, in the absence of SCPs, prominent motor and sensory neuronal death is observed [34,45-47].

\section{Myelination}

It has long been observed that in addition to the requirement of axons for the maintenance of a myelinating SC phenotype, the axon diameter determines whether an axon is myelinated and the thickness of the myelin sheath [48-50]. NRG1 is now known to be a key axon-derived factor in determining axon ensheathment and peripheral myelin thickness. Mice with a conditional ablation of erbB2 in SCs and double-heterozygote NRG1/erbB2 or triple-heterozygote NRG1/ erbB2/erbB3 mice all showed severe impairments in myelination, with thinner myelin sheaths [51,52]. NRG1 type III is the instructive isoform in PNS myelination: expression of this isoform in sympathetic ganglion cells that are normally unmyelinated can induce myelination. Mice that are haploinsufficient for NRG1 type III are hypomyelinated and mice overexpressing NRG1 type III (but not type I) have thicker myelin sheaths [52,53]. This NRG1 type III myelination signal is most potent when presented on the axonal surface acting in a juxtacrine manner; accessory signals on the axolemma are also required, as NRG1 type III expression on non-neuronal cells could not induce myelination [53]. Although NRG1 is most potent when presented in the cell membrane at certain low concentrations, soluble NRG1 isoforms (including types I, II and III) can have promyelinating effects in vitro $[53,54]$. To summarize the in vivo evidence, the determination of myelin thickness by NRG1 is dependent on the amount of NRG1 type III expressed on the axonal surface [53]. NRG1 expression on small-diameter axons is also required for the normal ensheathment of these axons by nonmyelinating SCs (Figure 2) [55,56]. Deficiency of NRG1 on the axolemma of small-diameter DRG cells results in enlarged Remak bundles with very little SC cytoplasm separating the axons and impaired sensory function [56].

\section{NRG1 signaling in SCs}

There have been significant advances in the understanding of signaling pathways regulating SC phenotypes by NRG1. Figure 1 shows a schematic view of NRG1-mediated signaling pathways in SCs. The binding of NRG1 to erbB2/3 heteromers on SCs results in tyrosine phosphorylation, the recruitment of adaptor molecules and the activation of a number of canonical signaling pathways. Downstream signaling pathways that transduce the essential NRG1 signal during myelination include the PI3K-Akt pathway, ERK1/2-MAPK and calcineurin-NFAT pathways, as well as the small GTPases Cdc42 and Racl and the nonreceptor tyrosine kinases FAK and SRC (Figure 1) [53,57-60]. However, the distinct roles and mechanisms of differential activation of these pathways are still unknown. One important theme to emerge is that NRG1 can have different roles at different stages of SC development. For instance, NRG1 promotes both SC proliferation (which is observed in the late embryonic/early postnatal period) and axon ensheathment and myelination in the postnatal period, a process that is incompatible with SC proliferation. This functional diversity is achieved through the interaction of NRG1 with other signaling pathways. One example is Notch signaling, which promotes the 
survival and proliferation of SCPs, increases the expression of erbB2 in SCPs and enhances their sensitivity to NRG1 [61]. At later stages of SC development, in order for myelination to proceed, there is an important interaction between cAMP and NRG1 signaling (see later) [62].

It was previously thought that the promotion of myelination by NRG1 type III/erbB signaling was mediated primarily via the PI3K/Akt pathway. The MAPK pathway was thought to have negative and opposing functions on myelination [50]. Axonal NRG1 type III was found to specifically activate the PI3K pathway [53]. PI3K activation promotes $\mathrm{SC}$ differentiation and myelin formation in SC-DRG co-cultures, and in vivo transfection with a mutant activated form of the downstream effector Akt was also shown to promote myelin formation in regenerating fibers [63]. Disrupting P13K downstream signaling results in inhibition of axonal-mediated SC proliferation and myelination in vitro [50], whereas activation of the MAPK, ERK by NRG1 inhibited myelin gene expression and myelination in vitro $[54,63]$. Recent findings suggest that the situation is more complex and, in fact, ERK-MAPK signaling is required for NRG1 signaling. Gene ablation of either $\operatorname{Shp} 2$ (which acts to promote sustained activation of this pathway) or ERK1 and ERK2 in SCs demonstrate that this pathway is essential in the NRG1-mediated survival of SCP and at later stages of development for myelination [64,65].

Calcineurin-NFAT signaling has also been shown to positively regulate myelination and is regulated by NRG1. Calcineurin is a phosphatase activated by $\mathrm{Ca}^{2+}$, leading to dephosphorylation and subsequent activation of NFAT transcription factors. Conditional mutation of calcineurin- $\beta 1$ in the neural crest results in severely disrupted peripheral myelin and a decrease in the expression of myelin-related proteins Krox 20, MAG, MBP and P0 [59]. NRG1 signaling stimulates the calcineurin-NFAT pathway by inducing phospholipase C- $\gamma$-dependent $\mathrm{Ca}^{2+}$ influx [59].

Schwann cell myelination is dependent on a transcriptional program. A number of transcription factors such as Oct6, NF- $\kappa B$ and Krox 20 are positive regulators of myelination, as their activity leads to the expression of myelin proteins including $\mathrm{P} 0, \mathrm{MBP}, \mathrm{PMP} 22$, connexin 32 and the lipid biosynthesis enzyme HMG-CoA [66]. Krox 20 is known as a master regulator of myelination. NRG1 increases the expression of this transcription factor via ERK-dependent phosphorylation of Yyl [67]. This is also an illustration of cooperation between NRG1 and another axon-derived pro-myelin signal. An as-yet unidentified axonal ligand activates GPR 126, which is expressed on SCs, leading to increased cAMP, which is critical for myelination [68]. NRG1-induced expression of Krox 20 and myelin-related proteins in mouse SCs requires the presence of elevated cAMP [62]. Crucially, cAMP appears to switch NRG1 from a proliferative signal to a myelin differentiation signal [62]. A further example of interaction between these pathways is that type III NRG1 and cAMP-dependent PKA phosphorylation act cooperatively to increase the activity of NF- $\mathrm{KB}$, which promotes myelination [69,70].

A nuclear-localized form of erbB3 (nuc-erbB3) has recently been described, the expression of which is regulated by the addition of exogenous NRG1. Nuc-erbB3 is expressed in postnatal SCs in rats, but is not detected in adulthood, suggesting developmental regulation. Nuc-erbB3 associates with genomic regions including promoters that regulate the expression of proteins involved in several SC functions, including radial sorting of axons and myelination. Inhibition of nuc-erbB3 reduces myelination in SC-neuron co-cultures. Therefore, it is possible that conventional NRG1/erbB signaling in SCs can drive the expression of an alternatively spliced transcript of the erbB3 gene, resulting in transcriptional regulation of the genes involved in myelination and other SC functions. The precise mechanisms of this pathway are yet to be determined [71].

A recent study sheds light on how myelination is terminated, an important issue given that excessive myelination is deleterious [72,73]. Given its importance as a promyelination signal, it is no surprise to find that there is a negative feedback loop acting on NRG1 signaling. As described earlier NRG1 signals via the PI3K-Akt pathway to initiate myelin production. Akt is inhibited by the activity of the enzyme PTEN. The importance of PTEN is illustrated by the fact that deletion of PTEN in SCs results in hypermyelination [74]. NRG1 signaling increases the abundance of Dlg1, which stabilizes PTEN reducing its proteosomal degradation, which reduces Akt activity and terminates myelination [73].

\section{The role of NRG1 in adulthood}

Because of its key developmental role, it has been difficult to study the function of endogenous NRG1 signaling in adulthood. There is now clear evidence that ongoing NRG1 signaling is not required for maintenance of the myelin sheath in adulthood. Conditional ablation of NRG1 in peripheral axons or the erbB2 receptor 
in SCs has no effect on axon or myelin integrity in naive adult nerves [75]. This is in contrast to Krox 20, which is essential for myelin maintenance in adulthood [76]. Although NRG1 is not required for myelin maintenance, there is increasing evidence that NRG1 does contribute to the response to nerve injury. This is complicated by the fact that NRG1 may have different roles during different phases of the injury response and acts not only as a signal between injured axons and SCs, but also between axons and macrophages and microglia.

\section{The expression of NRG1 isoforms \& their} receptors changes following nerve injury In SCs, the expression of Ig-containing isoforms of NRG1 and erbB2 and erbB3 receptors in the distal nerve significantly increases following sciatic nerve transection; this is first detected 3 days postinjury and is sustained for at least 30 days [77-79]. Guertin and coworkers examined the levels of erbB2 activation immediately following sciatic nerve transection and found that there is also an acute and transient increase in the levels of phosphorylated erbB2 and erbB3 in the first hour following injury, which decreases again by $3 \mathrm{~h}$ postinjury [80]. In chronically denervated sciatic nerves, expression levels of erbB receptors are much decreased [81].

From 3 days postinjury a sustained increase in erbB2 and erbB3 expression is seen, and activation of these receptors is indicated by enhanced phosphorylation of erbB2; this is the time at which SCs undergo injury-induced proliferation [79].The expression of NRG1 type III by peripheral neurons initially decreases following nerve injury and then increases as axons begin to re-innervate their targets [77,82]. Similar results were found in a facial nerve transection model where neuronal NRG1 type III levels were significantly downregulated and did not recover until 14 days postinjury [83]. In sural nerve biopsies from patients suffering from axonal neuropathies, increased levels of NRG1 $\beta$ type I have been reported in the early stages [84]. NRG1 expression was quantified in the target muscle flexor digitorum following denervation by median nerve transection. Denervation was found to dramatically increase the levels of NRG1 $\alpha$ isoforms and erbB2 and erbB3 receptors in the muscle and on re-innervation of the muscle the levels decreased [85].

The expression of NRG1 isoforms and erbB receptors is clearly dysregulated following peripheral nerve injury. This finding, along with the role of NRG1 in axoglial signaling during development, has led to the hypothesis that NRG1 may play a role in the response to nerve injury.

\section{Endogenous NRG1 may have a role in the early events of Wallerian degeneration}

Wallerian degeneration is the process by which transected axons and their associated myelin sheaths are removed [86]. Clearance of myelin debris is an important part of the regenerative response, as myelin contains axon outgrowth inhibitors and delayed myelin clearance is associated with impaired axon regeneration [87]. There is evidence that NRG1 may act as an early signal between damaged axons and SCs, promoting myelin breakdown [80]. Following axon transection, activation of erbB2 is observed within the paranodes of myelinating SCs. Rats treated with a pharmacological inhibitor PKI-166, which blocks the erbB2 receptor, results in a reduction in demyelination at 2 days post-nerve transection [80]. The role of NRG1 has not yet been conclusively demonstrated, as the inhibitor used acts on a broad range of erbB receptors including erbB1 (the EGF receptor), and so a number of ligands (including NRG1) may be implicated.

\section{Endogenous NRG1 is required for nerve repair}

For effective nerve repair, injured axons need to regenerate through an injured nerve, recontact their target organ, such as skin or muscle, and re-establish their normal relationship with SCs such that large-diameter axons are remyelinated. Using a transgenic system in which a small subpopulation of axons lacked NRG1 in adulthood demonstrated that such axons showed slowed axon regeneration measured at 10 and 14 days following sciatic nerve crush. At 8 weeks postinjury, axon outgrowth was equal to control. Because only a small proportion of axons (4\%) failed to express NRG1, this implicated juxtacrine NRG1 signaling in axon regeneration following nerve injury [88]. It is currently unclear as to the reason for the slowed rate of axon regeneration: in the absence of NRG1, less pro-regenerative factors such as neurotrophins may be produced by SCs [55]. Alternatively, NRG1 may act in a cell-autonomous manner to promote axon outgrowth, as observed in some CNS neurons [89].

Remyelination of regenerating axons is essential for the restoration of saltatory conduction. Axons lacking NRG1 were seen to have a gross impairment in remyelination following injury, with $38.5 \pm 3.3 \%$ having completely failed to elaborate 
a myelin sheath 8 weeks following sciatic nerve crush; axons that had remyelinated were hypomyelinated compared with controls (Figure 3) [88]. In a further study in which NRG1 was ablated in all peripheral axons, impaired remyelination was associated with slowed conduction velocity in regenerated axons, as well as impaired functional recovery following sciatic crush [90]. Indirectly, a role of NRG1 signaling in remyelination has also been implicated in a study looking at myelin thickness following sciatic nerve crush in mice lacking BACE1 [29]. In these mice, remyelination is delayed, myelin thickness is reduced and there is an increase in axons that fail to be remyelinated. BACE1 is known to cleave NRG1 type III and is required for normal myelination during development $[25,26]$. Therefore, correct processing of NRG1 may be required for effective remyelination following peripheral nerve injury. Recently, mice lacking BACE1 have been shown to have accelerated axonal and myelin debris clearance during Wallerian degeneration, accelerated axon regeneration and target re-innervation and increased neurite growth in culture [91]. This effect was independent of SC BACE1 expression and therefore likely to be primarily mediated by axonal and macrophage BACE1 expression. The mechanism for this enhanced regenerative response in the absence of BACE1 is yet to be determined.

Motoneurons lacking NRG1 demonstrate slowed re-innervation of their synaptic target, the neuromuscular junction. NRG1-deficient axons also showed excess terminal sprouts at 8 weeks following sciatic nerve crush [88]. It is possible that the increased terminal sprouting at the neuromuscular junction is not a direct consequence of a lack of NRG1, but a consequence of conduction block due to the impaired remyelination seen in these axons.

\section{Treatment with NRG1 can promote nerve repair}

Endogenous NRG1 clearly has a role in nerve repair, and there are a number of studies suggesting that application of exogenous NRG1 may promote recovery from nerve injury. Rats treated every $24 \mathrm{~h}$ for 4 days beginning $24 \mathrm{~h}$ after sciatic nerve injury with recombinant NRG1 type II showed improvement in measures of axon regeneration, including axon diameter and myelin thickness, assessed at 2 months postinjury, and had accelerated functional recovery as determined by the sciatic functional index (SFI) [92]. In addition, in rabbits with a facial nerve transection, treatment with NRG1 type II at the time of injury and 24 and $28 \mathrm{~h}$ postinjury resulted in an increase in SC nuclei and a reduction in myelin debris at 2 months postinjury [93]. Furthermore, rats injected intraneurally into both the proximal and distal stump of the sciatic nerve at the time of transection with an adenovirus expressing just the $\beta$-EGF domain of NRG1 type I increased the length of regenerating axons, expression of GAP43 at 3 weeks following injury and showed an improved functional outcome determined by gait analysis [94].
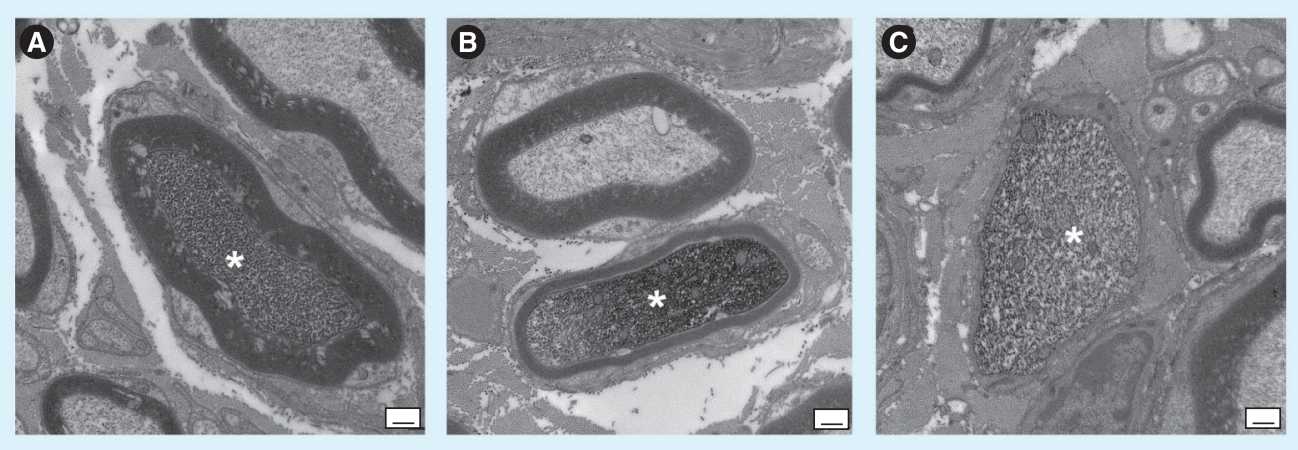

Figure 3. Neuregulin-1 is essential for remyelination following peripheral nerve injury. Electron micrographs of transverse sections of the sciatic nerve 8 weeks post-sciatic nerve crush injury in SLICK-A Cre;NRG1f/f mice in which neuregulin-1 (NRG1) is inducibly ablated in adulthood in a small percentage of axons that are labeled with yellow fluorescent protein (YFP) (B \& C) and control (A). YFP-expressing axons are identified using immunoelectron microscopy and the resulting presence of a dark reaction product (asterisks). Control animals demonstrated effective remyelination of YFPexpressing axons (A). By contrast, YFP-positive axons from conditional NRG1-mutant (tamoxifentreated SLICK-A Cre;NRG1fl/fI) mice either had a significantly thinner myelin sheath (B) or completely failed to elaborate a myelin sheath (C). Note that in (B), a neighboring axon that is YFP negative is normally myelinated, highlighting the importance of juxtacrine NRG1 signaling in remyelination following peripheral nerve injury. Scale bars: $500 \mathrm{~nm}$.

Reproduced with permission from [88]. 
These studies imply that NRG1 signaling may have a role in peripheral regeneration and provide some hope that treatment with recombinant forms of Ig-NRG1s may improve the outcome following peripheral nerve injury. NRG1 also has a role in cardiac maintenance and indeed clinical trials are underway for its use in heart failure [95]. The potential mechanisms of how NRG1 signaling may modulate nerve repair have not been extensively studied; these will be discussed in more detail later. A caveat of administering recombinant NRG1 protein is that enhanced myelination is best achieved by juxtacrine signaling in the cell membrane and, furthermore, excessive NRG1 signaling may be deleterious.

\section{Potential mechanisms of action of NRG1 signaling in peripheral nerve injury}

Neuregulin-1 signaling has been shown to be critical in axoglial signaling between peripheral neurons and SCs. In vitro investigations into the potential roles of NRG1 in SC function have been extensive and have focussed on survival, proliferation and migration of SCs (discussed earlier in the context of development). Several studies have looked at this using in vitro assays that attempt to recapitulate a nerve injury situation.

Recombinant NRG1 type II has been shown to influence the motility of SCs in DRG explants cultured on cryosections of peripheral nerve and in a superior cervical ganglion explant culture system designed to replicate the entubulation repair of transected peripheral nerves [96]. In agreement with this, treatment of chronically denervated nerve explants with recombinant NRG1 type II in the presence of forskolin increased the number of SCs migrating from the explants [97]. Furthermore, cultured peritoneal macrophages express erbB2, erbB3 and, erbB 4 and in response to recombinant NRG1 $\beta$-EGF domain, show enhanced motility (Figure 4) [98]. Therefore, in the context of peripheral nerve injury, there is some in vitro evidence that NRG1 signaling may play a role in the migration of both SCs and macrophages. Potentially, the expression/release of NRG1 by SCs and axons acts as a chemoattractant to SCs and macrophages in the initial stage of Wallerian degeneration. Figure 5 is a schematic diagram showing some of the potential roles of NRG1 at different stages of nerve repair.

Treatment of SC neuronal co-cultures with recombinant NRG1 type II has been shown to dose-dependently stimulate proliferation of SCs $[96,97]$. The use of a pharmacological inhibitor of all erbB receptors produced a reduction in SC proliferation 2 days after sciatic nerve axotomy [80]. By contrast transgenic mice with an inducible erbB2 ablation in adult SCs did not show any change in the level of SC proliferation at either 4 or 12 days post-sciatic nerve transection [75]. Therefore, the role of NRG1 signaling in the proliferative response of SCs following nerve injury has not yet been fully resolved; one possible reason for these contrasting results is that

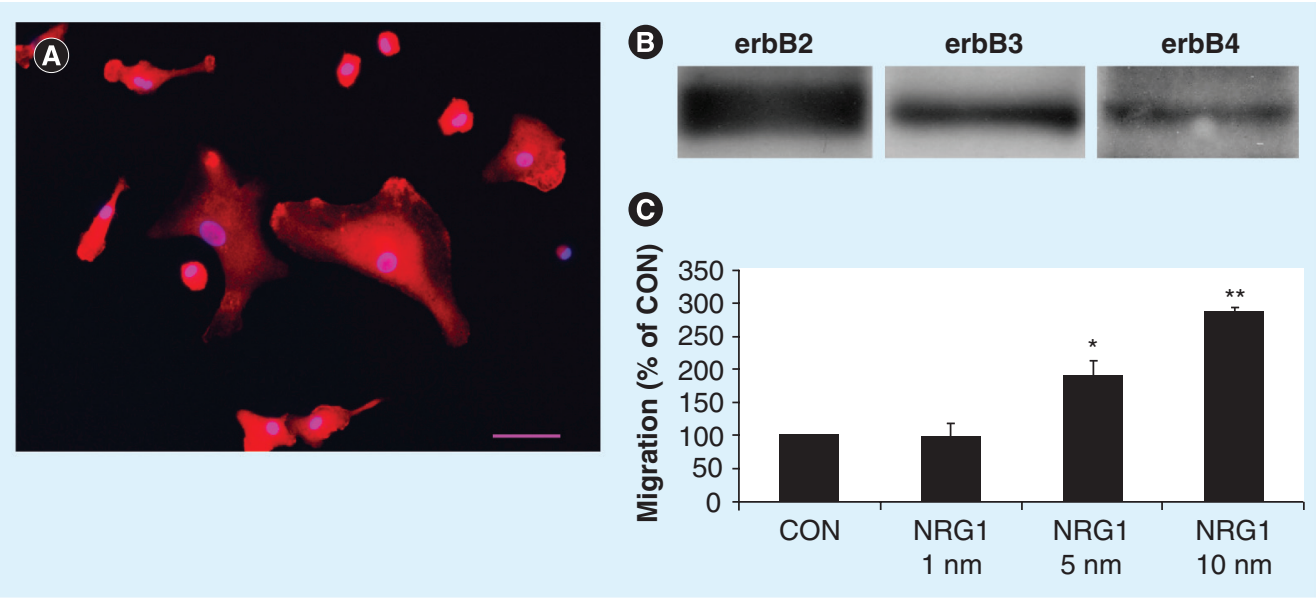

Figure 4. Macrophages express erbB2, erbB3 and erbB4 and neuregulin-1 enhances macrophages motility. Peritoneal macrophages were cultured, these cells are identified by IBA1 immunostaining and nuclear staining with DAPI (A). These cells expressed erbB2, erbB3 and erbB4 (185-kDa band in western blot) (B) and showed enhanced motility in response to NRG1 assessed in the Boyden chamber assay $(\mathbf{C})$. Error bars represent \pm standard error of the mean (three independent experiments). The statistical tests used were one-way analysis of variance with Bonferroni post hoc analysis. Scale bar: $20 \mu \mathrm{m} .{ }^{*} \mathrm{p}<0.05,{ }^{*} \mathrm{p}<0.001$.

CON: Control; NRG1: Neuregulin-1.

Reproduced with permission from [98]. 


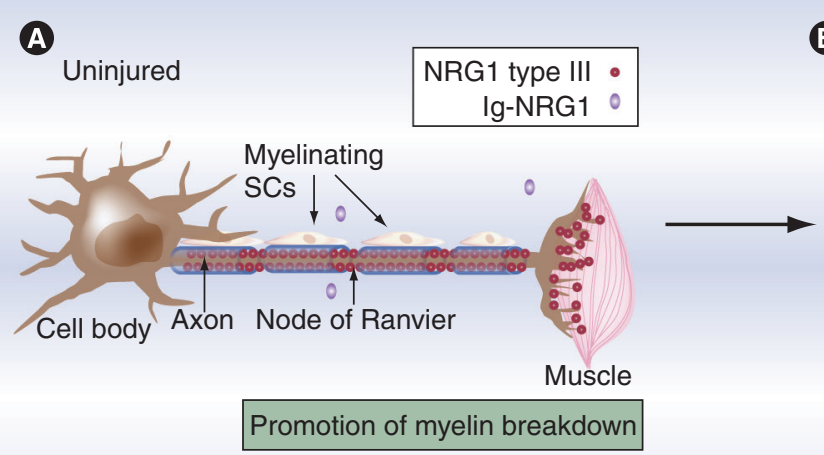

B Following injury, SCs dedifferentiate and proliferate

D

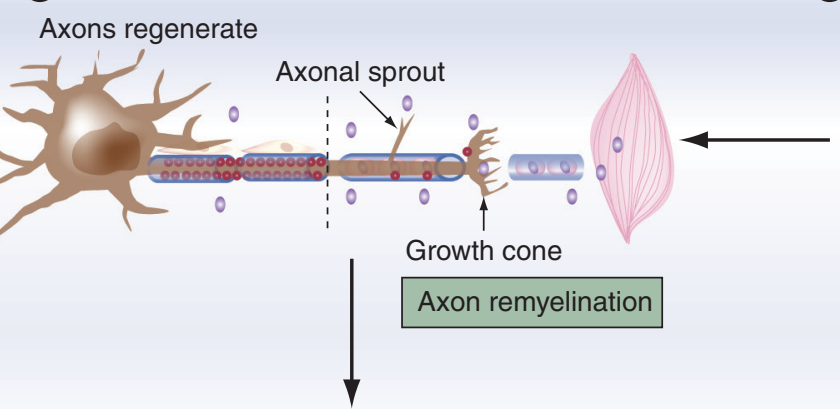

Transection Myelin/axonal

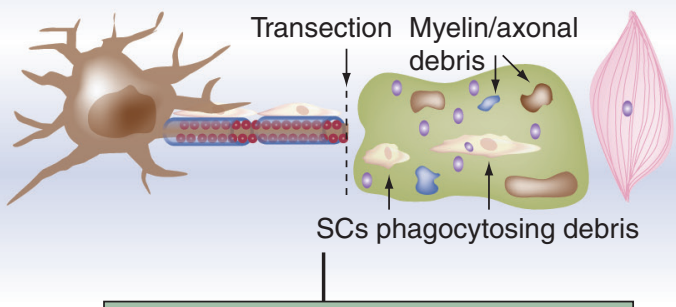

Enhancing macrophage and SC migration

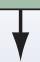

C Macrophages infiltrate and phagocytose debris

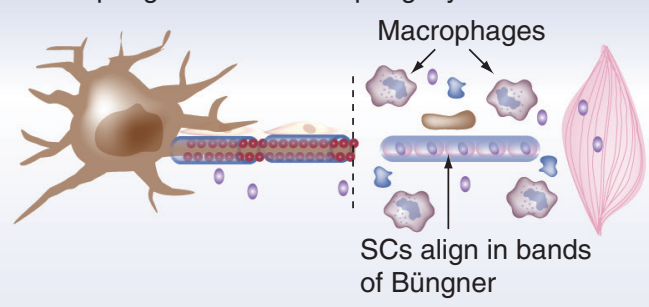

E

SC survival and axon regeneration

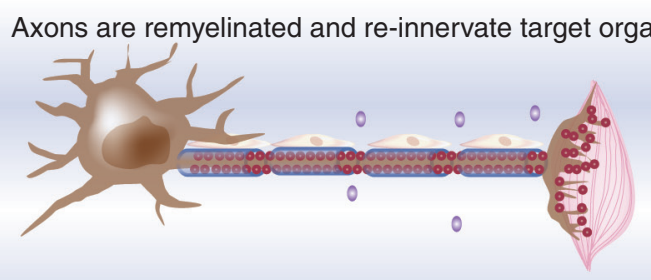

Future Neurol. ๑ Future Science Group (2011)

Figure 5. Neuregulin-1 is potentially involved at multiple stages of the nerve repair process following injury. Events after a peripheral nerve injury and the potential roles NRG1 may play in this process (dark boxes) (A-E). Following peripheral nerve injury specifically a transection injury - a series of regulated events occur termed Wallerian degeneration. (B) Within 2 days following injury, the distal stump fragments and SCs begin clearance of axonal and myelin debris, and at the same time SCS proliferate and dedifferentiate. NRG1 has been implicated in promotion of this initial myelin clearance [80]. During the acute phase following injury, the expression levels of Ig-NRG1s, erbB2 and erbB3 increase in SCs, and this expression is sustained long after myelin clearance. Neuronal expression of NRG1 type III decreases and does not return to normal levels until axons re-innervate their target organs. (C) Macrophages infiltrate into the distal stump, and within 2 weeks, both macrophages and SCs complete the clearance of debris, and at the same time, dedifferentiated SCS align themselves in bands of Büngner. NRG1 is known to enhance macrophage motility, so it may play a role in macrophage recruitment following nerve injury, although this has not yet been shown in vivo [98]. (D) Axons then regenerate out of the proximal stump following SC tubes and remake contact with SCS. NRG1 is known to play a role in axon regeneration [88] and may also have a role in SC survival, particularly in chronically denervated nerve stumps. (E) Axons re-innervate their target organs and are remyelinated by SCs in order to regain full function. NRG1 is known to be essential for remyelination of axons following nerve injury [88]. NRG1 may therefore be involved at multiple stages of peripheral nerve repair.

Ig: Immunoglobulin; NRG1: Neuregulin-1; SC: Schwann cell.

the pharmacological inhibitor used will not only block NRG1 signaling, but also a wide range of EGFR ligands.

A further potential mechanism of action of NRG1 following nerve injury is in the survival of SCs, a role that well characterized in development. In the postnatal period in rodents, denervation induces apoptosis of both terminal SCs associated with neuromuscular junctions and SCs associated with the mechanoreceptors,
Golgi tendon organs and Pacinian corpuscles. Treatment with recombinant NRG1 type II can improve survival of these populations of SCs [99,100]. Similarly, postnatal SCs in the sciatic nerve were protected from axotomy-induced apoptosis on treatment with recombinant NRG1 type II [101]. However, in adult animals, denervation or axotomy does not induce apoptosis in these SCs, highlighting the different survival requirements of SCs throughout development, 
and consequently the survival function of NRG1 type II may not be applicable in an adult injury state. After chronic denervation, a progressive loss of SCs is seen both in animals and humans [102]. Given the critical role of these cells for axon regeneration, this is likely to significantly impair functional recovery. There is evidence that chronically denervated SCs can still respond to NRG1 in vitro [97]. If NRG1 could provide a means to maintain SCs in a healthy state until they re-establish a relationship with axons, this could provide a means to promote functional recovery, particularly after proximal lesions such as brachial plexus injuries.

Neuregulin-1 is obviously a key axoglial signal for myelination. In many traumatic neuropathies, although remyelination is associated with shorter internodal length and a larger g-ratio [103,104], saltatory conduction is effectively restored, and there is only a small reduction in conduction velocity. In certain hereditary neuropathies such as CharcotMarie-Tooth type I and immune-mediated neuropathies such Guillain-Barré syndrome, demyelination is much more of a problem and, over a long period of time, may lead to secondary axonal dysfunction. It is an as-yet unanswered question whether in a primary demyelinating disorder NRG1 could promote remyelination. NRG1 may also have either direct or indirect effects on axonal protection in disease states.

\section{Too much of a good thing? Excess NRG1 signaling may be harmful}

Excessive stimulation of NRG1 signaling may be detrimental for nerve function, an issue that could have important implications for any therapeutic use of the molecule if the therapeutic window is narrow. Transgenic mice that overexpress NRG1 type II- $\beta 3$ in myelinating SCs (onset of expression at postnatal day 5) develop hindlimb paralysis, evidence of peripheral nerve demyelination and remyelination and, by 6-7 months of age, these animals developed malignant peripheral nerve sheath tumors [105], implying that excessive constitutive activation of NRG1 type II- $\beta 3$ signaling induces aberrant $S C$ activity. Although low doses of soluble NRG1 can promote myelination in vitro [54], high doses of NRG1 have been reported to cause demyelination $[80,106]$. Leprosy is a leading cause of neuropathy in the developing world. Mycobacterium leprae binds to and activates the erbB2 receptor, bypassing the requirement for erbB2-erbB3 heterodimerization. This results in strong activation of the MEK-ERK pathway and subsequently demyelination. In this context, inhibition of erbB2 signaling could abrogate the demyelination caused by $M$. leprae. These points illustrate the fact that excessive activation of erbB2 receptors on SCs can be harmful [107].

\section{Conclusion \& future perspective}

In relation to NRG1 biology, we now have a much more complete understanding of which signaling pathways are activated by the binding of this molecule to erbB $2 / 3$ receptors on SCs. There is still a great deal to learn about how these signaling systems interact and how NRG1 may produce different outcomes depending on the state of SC differentiation. Different NRG1 isoforms differentially activate such signaling pathways, and the mechanism of such differential activation is poorly understood. It may relate to the kinetics of receptor binding and/or the pattern of receptor tyrosine phosphorylation and hence recruitment of adaptor proteins. Given the role of NRG1 in peripheral nerve repair, an important objective is to see if this pathway can be manipulated in order to promote restoration of function after nerve injury. There is some encouraging data in animal models, and human trials using soluble NRG1 isoforms are underway for the treatment of heart failure [95]. Consideration will need to be given to the exact isoform of NRG1 used, but also to how it is presented to the SC. Most current data would suggest that to promote remyelination, NRG1 would be most effective if present in the axolemma, which would necessitate the use of a viral vector to express a type III isoform, a technique that is still in its infancy in humans. Soluble NRG1 can promote myelination; however, this is likely to have a narrower therapeutic index. The effects of NRG1 may not be restricted to remyelination: there is evidence that it can promote axon outgrowth, and it would be very interesting to know if it could increase SC survival after prolonged denervation. Therapeutic strategies need not be restricted to the use of NRG1 itself; for instance, the protease TACE (ADAM17) cleaves NRG1, reducing its activity, and so inhibition of TACE would be predicted to enhance NRG1 activity and hence increase remyelination [27]. It is our hope that greater understanding of this key axoglial signaling pathway that is required for peripheral nerve development will lead to future novel strategies for peripheral nerve repair. 


\section{Executive summary}

\section{Peripheral nerve injury}

- Peripheral nerve injury causes significant morbidity. Current surgical repair approaches are inadequate and as yet there are no pharmacological therapies that enhance peripheral nerve repair.

\section{Neuregulins \& the PNS}

- Neuregulins (NRGs) are a family of growth factors (NRG1-4). NRG1 has many isoforms characterized by their N-terminal domains into types I-VI, all of which signal through homo- and hetero-dimers of the tryrosine kinase receptors erbB2, erbB3 and erbB4.

- NRG1 is known to be an important signaling factor at virtually all stages of Schwann cell (SC) development, from migratory neural crest cells to formation of mature SCs.

- NRG1-erbB2/3 signaling between axons and SCs is essential for PNS myelination. Critically, the level of NRG1 type III expressed on the axon surface determines the ensheathment fate of an axon and the thickness of the myelin sheath.

- NRG1 is also important for correct ensheathment of small unmyelianed axons by non-myelinating SCs.

\section{NRG1 signaling in SCs}

- NRG1/erbB2/3 signaling in SCs transduces the PI3K-Akt, ERK1/2-MAPK and calcineurin-NFAT pathways, as well as the small GTPases Cdc42 and Rac1 and the nonreceptor tyrosine kinases FAK and SRC.

\section{The role of NRG1 in adulthood}

- NRG1 is not required for myelin maintenance in adulthood but is involved in the repair process following nerve injury.

- Following peripheral nerve injury, the expression of erbB2 and erbB3 receptors and immunoglobulin NRG1 isoforms are rapidly increased.

- NRG1 signaling may be involved in the early myelin clearance stage of Wallerian degeneration.

- In the absence of juxtacrine NRG1 signaling, there is impairment in axon regeneration and remyelination following peripheral nerve injury, highlighting NRG1's role in nerve repair.

- Several studies indicate that treatment with recombinant immunoglobulin NRG1 can promote nerve repair.

\section{Potential mechanisms of action of NRG1 signaling in peripheral nerve injury}

- Potential mechanisms of NRG1 action during peripheral nerve repair include migration of SCs and macrophages, SC survival and remyelination of axons.

- Excessive stimulation of the NRG1-erbB2 pathway may be harmful, an example of which is seen in the demyelinating neuropathy caused by Mycobacterium leprae binding to and activating erbB2 in leprosy.

\section{Future perspective}

- Data suggest that NRG1 signaling plays a role in repair following peripheral nerve injury.

- Manipulation of NRG1 signaling may provide a mechanism of promoting axon regeneration and remyelination.

- Current data suggest the most effective approach to improve remyelination may be to enhance NRG1 type III presentation on the axolemma, necessitating the use of a viral vector expressing NRG1 type III. Further options would be to manipulate downstream signaling pathways in SCS or NRG1 processing (e.g., inhibition of TNF- $\alpha$-converting enzyme).

\section{Financial \& competing interests disclosure}

FR Fricker is a CASE PhD student funded by Acorda Therapeutics, Inc., and the Biotechnology and Biological Sciences Research Council (BBSRC). DLH Bennett would like to acknowledge the financial support of the Wellcome trust and the BBSRC. The authors have no other relevant affiliations or financial involvement with any organization or entity with a financial interest in or financial conflict with the subject matter or materials discussed in the manuscript apart from those disclosed.

No writing assistance was utilized in the production of this manuscript.

\section{Bibliography}

Papers of special note have been highlighted as: - of interest

." of considerable interest
1. Martyn CN, Hughes RA. Epidemiology of peripheral neuropathy. J. Neurol. Neurosurg. Psychiatry. 62(4), 310-318 (1997).

2. Mohanna PN, Young RC, Wiberg M, Terenghi G. A composite poly-hydroxybutyrateglial growth factor conduit for long nerve gap repairs. J. Anat. 203(6), 553-565 (2003).

3. Filbin MT. Myelin-associated inhibitors of axonal regeneration in the adult mammalian CNS. Nat. Rev. Neurosci. 4(9), 703-713 (2003).

4. Matsuyama T, Mackay M, Midha R. Peripheral nerve repair and grafting techniques: a review. Neurol. Med. Chir. 40(4), 187-199 (2000).

5. Webber C, Zochodne D. The nerve regenerative microenvironment: early behavior and partnership of axons and Schwann cells. Exp. Neurol. 223(1), 51-59 (2010).

6. Zhang D, Sliwkowski MX, Mark M et al. Neuregulin-3 (NRG3): a novel neural tissue-enriched protein that binds and activates erbB4. Proc. Natl Acad. Sci. USA 94(18), 9562-9567 (1997).
7. Harari D, Tzahar E, Romano J et al. Neuregulin-4: a novel growth factor that acts through the erbB-4 receptor tyrosine kinase. Oncogene 18(17), 2681-2689 (1999).

8. Marchionni MA, Goodearl AD, Chen MS et al. Glial growth factors are alternatively spliced erbB2 ligands expressed in the nervous system. Nature 362(6418), 312-318 (1993).

9. Carraway KL 3rd, Weber JL, Unger MJ et al. Neuregulin-2, a new ligand of erbB3/ erbB4-receptor tyrosine kinases. Nature 387(6632), 512-516 (1997).

10. Lemke G. Neuregulins in development. Mol. Cell Neurosci. 7(4), 247-262 (1996).

11. Falls DL, Rosen KM, Corfas G, Lane WS, Fischbach GD. ARIA, a protein that stimulates acetylcholine receptor synthesis, is a member of the neu ligand family. Cell 72(5), 801-815 (1993). 
12. Goodearl AD, Davis JB, Mistry Ket al. Purification of multiple forms of glial growth factor. J. Biol. Chem. 268(24), 18095-18102 (1993).

13. Holmes WE, Sliwkowski MX, Akita RW et al. Identification of heregulin, a specific activator of p185erbB2. Science 256(5060), 1205-1210 (1992).

14. Peles E, Bacus SS, Koski RA et al. Isolation of the neu/HER-2 stimulatory ligand: a $44 \mathrm{kd}$ glycoprotein that induces differentiation of mammary tumor cells. Cell 69(1), 205-216 (1992).

15. Falls DL. Neuregulins: functions, forms, and signaling strategies. Exp. Cell Res. 284(1), 14-30 (2003).

16. Mei L, Xiong WC. Neuregulin 1 in neural development, synaptic plasticity and schizophrenia. Nat. Rev. Neurosci. 9(6), 437-452 (2008).

17. Shamir A, Buonanno A. Molecular and cellular characterization of neuregulin-1 type IV isoforms. J. Neurochem. 113(5), 1163-1176 (2010).

18. Liu X, Bates R, Yin DM et al. Specific regulation of NRG1 isoform expression by neuronal activity. J. Neurosci. 31(23), 8491-8501 (2011).

19. Li Q, Loeb JA. Neuregulin-heparan-sulfate proteoglycan interactions produce sustained erbB receptor activation required for the induction of acetylcholine receptors in muscle. J. Biol. Chem. 276(41), 38068-38075 (2001).

20. Loeb JA, Fischbach GD. ARIA can be released from extracellular matrix through cleavage of a heparin-binding domain. J. Cell Biol. 130(1), 127-135 (1995).

21. Wang JY, Miller SJ, Falls DL. The $\mathrm{N}$-terminal region of neuregulin isoforms determines the accumulation of cell surface and released neuregulin ectodomain. J. Biol. Chem. 276(4), 2841-2851 (2001).

22. Shirakabe K, Wakatsuki S, Kurisaki T, Fujisawa-Sehara A. Roles of meltrin $\beta$ / ADAM19 in the processing of neuregulin. J. Biol. Chem. 276(12), 9352-9358 (2001).

23. Yokozeki T, Wakatsuki S, Hatsuzawa K, Black RA, Wada I, Sehara-Fujisawa A. Meltrin $\beta$ (ADAM19) mediates ectodomain shedding of neuregulin $\beta 1$ in the Golgi apparatus: fluorescence correlation spectroscopic observation of the dynamics of ectodomain shedding in living cells. Genes Cells 12(3), 329-343 (2007).

24. Luo X, Prior M, He W et al. Cleavage of neuregulin-1 by BACE1 or ADAM10 produces differential effects on myelination. J. Biol. Chem. 286(27), 23967-23974 (2011).
25. Hu X, Hicks CW, He W et al. BACE1 modulates myelination in the central and peripheral nervous system. Nat. Neurosci. 9(12), 1520-1525 (2006).

- Pro-neuregulin-1 (NRG1) type III is cleaved by $\beta$-secretase $\beta$-site of amyloid precursor protein-cleaving enzyme (BACE) and this is required for full activity of the molecule.

26. Willem M, Garratt AN, Novak B et al. Control of peripheral nerve myelination by the $\beta$-secretase BACE1. Science 314(5799), 664-666 (2006).

- Pro-NRG1 type III is cleaved by BACE and this is required for full activity of the molecule.

27. La Marca R, Cerri F, Horiuchi K et al. TACE (ADAM17) inhibits Schwann cell myelination. Nat. Neurosci. 14(7), 857-865 (2011).

-. Interestingly, pro-NRG1 type III can be cleaved by TNF- $\alpha$-converting enzyme at a different position to BACE, and this reduces activity of the molecule.

28. Ohno M, Hiraoka Y, Matsuoka T et al. Nardilysin regulates axonal maturation and myelination in the central and peripheral nervous system. Nat. Neurosci. 12(12), 1506-1513 (2009).

29. $\mathrm{Hu} X, \mathrm{He} W$, Diaconu $\mathrm{C}$ et al. Genetic deletion of $B A C E 1$ in mice affects remyelination of sciatic nerves. FASEB J. 22(8), 2970-2980 (2008).

30. Roskoski R Jr. The erbB/HER receptor protein-tyrosine kinases and cancer. Biochem Biophys. Res. Commun. 319(1), 1-11 (2004).

31. Yarden Y, Sliwkowski MX. Untangling the erbB signalling network. Nat. Rev. Mol. Cell Biol. 2(2), 127-137 (2001).

32. Meyer D, Yamaai T, Garratt A et al. Isoformspecific expression and function of neuregulin. Development 124(18), 3575-3586 (1997).

33. Woodhoo A, Sommer L. Development of the Schwann cell lineage: from the neural crest to the myelinated nerve. Glia 56(14), 1481-1490 (2008).

34. Jessen KR, Mirsky R. The origin and development of glial cells in peripheral nerves. Nat. Rev. Neurosci. 6(9), 671-682 (2005).

-. Detailed review of axoglial signaling during development of the PNS.

35. Britsch S, Goerich DE, Riethmacher D et al. The transcription factor Sox 10 is a key regulator of peripheral glial development. Genes Dev. 15(1), 66-78 (2001).

36. Riethmacher D, Sonnenberg-Riethmacher E, Brinkmann V, Yamaai T, Lewin GR, Birchmeier C. Severe neuropathies in mice with targeted mutations in the erbB3 receptor. Nature 389 (6652), 725-730 (1997).
37. Lee KF, Simon H, Chen H, Bates B, Hung MC, Hauser C. Requirement for neuregulin receptor erbB2 in neural and cardiac development. Nature 378(6555), 394-398 (1995).

38. Meyer D, Birchmeier C. Multiple essential functions of neuregulin in development. Nature 378 (6555), 386-390 (1995).

39. Morris JK, Lin W, Hauser C, Marchuk Y, Getman D, Lee KF. Rescue of the cardiac defect in erbB2 mutant mice reveals essential roles of erbB2 in peripheral nervous system development. Neuron 23(2), 273-283 (1999).

40. Woldeyesus MT, Britsch S, Riethmacher D et al. Peripheral nervous system defects in erbB2 mutants following genetic rescue of heart development. Genes Dev. 13(19), 2538-2548 (1999).

41. Wolpowitz D, Mason TB, Dietrich P, Mendelsohn M, Talmage DA, Role LW. Cysteine-rich domain isoforms of the neuregulin-1 gene are required for maintenance of peripheral synapses. Neuron 25(1), 79-91 (2000).

42. Meintanis S, Thomaidou D, Jessen KR, Mirsky R, Matsas R. The neuron-glia signal $\beta$-neuregulin promotes Schwann cell motility via the MAPK pathway. Glia 34(1), 39-51 (2001).

43. Lyons DA, Pogoda HM, Voas MG et al. erbB3 and erbB2 are essential for Schwann cell migration and myelination in zebrafish. Curr. Biol. 15(6), 513-524 (2005).

44. Ma Z, Wang J, Song F, Loeb JA. Critical period of axoglial signaling between neuregulin-1 and brain-derived neurotrophic factor required for early Schwann cell survival and differentiation. J. Neurosci. 31(26), 9630-9640 (2011).

45. Dong Z, Brennan A, Liu N et al. Neu differentiation factor is a neuron-glia signal and regulates survival, proliferation, and maturation of rat Schwann cell precursors. Neuron 15(3), 585-596 (1995).

46. Dong Z, Sinanan A, Parkinson D, Parmantier E, Mirsky R, Jessen KR. Schwann cell development in embryonic mouse nerves. J. Neurosci. Res. 56(4), 334-348 (1999).

47. Maurel P, Salzer JL. Axonal regulation of Schwann cell proliferation and survival and the initial events of myelination requires PI 3-kinase activity. J. Neurosci. 20(12), 4635-4645 (2000).

48. Garbay B, Heape AM, Sargueil F, Cassagne C. Myelin synthesis in the peripheral nervous system. Prog. Neurobiol. 61(3), 267-304 (2000).

49. Voyvodic JT. Target size regulates calibre and myelination of sympathetic axons. Nature 342(6248), 430-433 (1989). 
50. Nave KA, Salzer JL. Axonal regulation of myelination by neuregulin 1. Curr. Opin. Neurobiol. 16(5), 492-500 (2006).

51. Garratt AN, Voiculescu O, Topilko P, Charnay P, Birchmeier C. A dual role of erbB2 in myelination and in expansion of the Schwann cell precursor pool. J. Cell Biol. 148(5), 1035-1046 (2000).

52. Michailov GV, Sereda MW, Brinkmann BG et al. Axonal neuregulin-1 regulates myelin sheath thickness. Science 304(5671), 700-703 (2004).

- Seminal paper characterizing multiple transgenic mice either overexpressing NRG1 isoforms or with disrupted NRG1-erbB signaling, showing the role of NRG1 type III in myelination of peripheral axons.

53. Taveggia C, Zanazzi G, Petrylak A et al. Neuregulin-1 type III determines the ensheathment fate of axons. Neuron 47(5), 681-694 (2005).

- Important paper characterizing NRG1 type III's role in axon ensheathment and myelination.

54. Syed N, Reddy K, Yang DP et al. Soluble neuregulin-1 has bifunctional, concentrationdependent effects on Schwann cell myelination. J. Neurosci. 30(17), 6122-6131 (2010).

55. Chen S, Rio C, Ji RR et al. Disruption of erbB receptor signaling in adult nonmyelinating Schwann cells causes progressive sensory loss. Nat. Neurosci. 6(11), 1186-1193 (2003).

56. Fricker FR, Zhu N, Tsantoulas $\mathrm{C}$ et al. Sensory axon-derived neuregulin-1 is required for axoglial signaling and normal sensory function but not for long-term axon maintenance. J. Neurosci. 29(24), 7667-7678 (2009).

57. Newbern JM, Li X, Shoemaker SE et al. Specific functions for ERK/MAPK signaling during PNS development. Neuron 69(1), 91-105 (2011).

58. Yamauchi J, Miyamoto Y, Chan JR, Tanoue A. ErbB2 directly activates the exchange factor Dock7 to promote Schwann cell migration. J. Cell Biol. 181(2), 351-365 (2008).

59. Kao SC, Wu H, Xie J et al. Calcineurin/ NFAT signaling is required for neuregulin-regulated Schwann cell differentiation. Science 323(5914), 651-654 (2009).

60. Grove M, Komiyama NH, Nave KA, Grant SG, Sherman DL, Brophy PJ. FAK is required for axonal sorting by Schwann cells. J. Cell Biol. 176(3), 277-282 (2007).
61. Woodhoo A, Alonso MB, Droggiti A et al. Notch controls embryonic Schwann cell differentiation, postnatal myelination and adult plasticity. Nat. Neurosci. 12(7), 839-847 (2009).

62. Arthur-Farraj P, Wanek K, Hantke J et al. Mouse Schwann cells need both NRG1 and cyclic AMP to myelinate. Glia 59(5), 720-733 (2011).

63. Ogata T, Iijima S, Hoshikawa $S$ et al. Opposing extracellular signal-regulated kinase and Akt pathways control Schwann cell myelination. J. Neurosci. 24(30), 6724-6732 (2004).

64. Newbern J, Birchmeier C. NRG1/erbB signaling networks in Schwann cell development and myelination. Semin. Cell Dev. Biol. 21(9), 922-928 (2010).

65. Grossmann KS, Wende H, Paul FE et al. The tyrosine phosphatase Shp2 (PTPN11) directs neuregulin-1/erbB signaling throughout Schwann cell development. Proc. Natl Acad. Sci. USA 106(39), 16704-16709 (2009).

- Recent publication detailing the importance of the ERK-MAPK signaling pathway during PNS development.

66. Svaren J, Meijer D. The molecular machinery of myelin gene transcription in Schwann cells. Glia 56(14), 1541-1551 (2008).

67. He Y, Kim JY, Dupree J et al. Yy1 as a molecular link between neuregulin and transcriptional modulation of peripheral myelination. Nat. Neurosci. 13(12), 1472-1480 (2010).

68. Monk KR, Naylor SG, Glenn TD et al. A G protein-coupled receptor is essential for Schwann cells to initiate myelination. Science 325(5946), 1402-1405 (2009).

69. Limpert AS, Carter BD. Axonal neuregulin 1

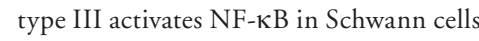
during myelin formation. J. Biol. Chem. 285(22), 16614-16622 (2010).

70. Yoon C, Korade Z, Carter BD. Protein kinase A-induced phosphorylation of the p65 subunit of nuclear factor- $\kappa \mathrm{B}$ promotes Schwann cell differentiation into a myelinating phenotype. J. Neurosci. 28(14), 3738-3746 (2008).

71. Adilakshmi T, Ness-Myers J, MadridAliste C, Fiser A, Tapinos N. A nuclear variant of erbB3 receptor tyrosine kinase regulates ezrin distribution and Schwann cell myelination. J. Neurosci. 31(13), 5106-5119 (2011).

72. Bolino A, Muglia M, Conforti FL et al. Charcot-Marie-Tooth type $4 \mathrm{~B}$ is caused by mutations in the gene encoding myotubularin-related protein-2. Nat. Genet. 25(1), 17-19 (2000).
73. Cotter L, Ozcelik M, Jacob C et al. Dlg1-PTEN interaction regulates myelin thickness to prevent damaging peripheral nerve overmyelination. Science 328(5984), 1415-1418 (2010).

74. Goebbels S, Oltrogge JH, Kemper R et al. Elevated phosphatidylinositol 3,4,5-trisphosphate in glia triggers cellautonomous membrane wrapping and myelination. J. Neurosci. 30(26), 8953-8964 (2010).

75. Atanasoski S, Scherer SS, Sirkowski E et al. ErbB2 signaling in Schwann cells is mostly dispensable for maintenance of myelinated peripheral nerves and proliferation of adult Schwann cells after injury. J. Neurosci. 26(7), 2124-2131 (2006).

76. Decker L, Smarquet-Trin-Dinh C, Taillebourg E, Ghislain J, Vallat JM, Charnay P. Peripheral myelin maintenance is a dynamic process requiring constant Krox 20 expression. J. Neurosci. 26(38), 9771-9779 (2006).

77. Carroll SL, Miller ML, Frohnert PW, Kim SS, Corbett JA. Expression of neuregulins and their putative receptors, erbB2 and erbB3, is induced during Wallerian degeneration. J. Neurosci. 17(5), 1642-1659 (1997).

78. Cohen JA, Yachnis AT, Arai M, Davis JG, Scherer SS. Expression of the neu protooncogene by Schwann cells during peripheral nerve development and Wallerian degeneration. J. Neurosci. Res. 31(4), 622-634 (1992).

79. Kwon YK, Bhattacharyya A, Alberta JA et al. Activation of erbB2 during Wallerian degeneration of sciatic nerve. J. Neurosci. 17(21), 8293-8299 (1997).

80. Guertin AD, Zhang DP, Mak KS, Alberta JA, Kim HA. Microanatomy of axon/glial signaling during Wallerian degeneration. J. Neurosci. 25(13), 3478-3487 (2005).

81. Li H, Terenghi G, Hall SM. Effects of delayed re-innervation on the expression of c-erbB receptors by chronically denervated rat Schwann cells in vivo. Glia 20(4), 333-347 (1997).

82. Bermingham-McDonogh O, Xu YT, Marchionni MA, Scherer SS. Neuregulin expression in PNS neurons. Isoforms and regulation by target interactions. Mol. Cell. Neurosci. 10(3-4), 184-195 (1997).

83. Kerber G, Streif R, Schwaiger FW, Kreutzberg GW, Hager G. Neuregulin-1 isoforms are differentially expressed in the intact and regenerating adult rat nervous system. J. Mol. Neurosci. 21(2), 149-165 (2003).

84. Oka N, Kawasaki T, Matsui M, Tachibana H, Sugita M, Akiguchi I. Neuregulin is associated with nerve regeneration in axonal neuropathies. Neuroreport 11(17), 3673-3676 (2000). 
85. Nicolino S, Panetto A, Raimondo S et al. Denervation and reinnervation of adult skeletal muscle modulate mRNA expression of neuregulin-1 and erbB receptors. Microsurgery 29(6), 464-472 (2009)

86. Coleman MP, Freeman MR. Wallerian degeneration, Wld(s), and NMNAT. Annu. Rev. Neurosci. 33, 245-267 (2010).

87. Barrette B, Hebert MA, Filali M et al. Requirement of myeloid cells for axon regeneration. J. Neurosci. 28(38), 9363-9376 (2008)

88. Fricker FR, Lago N, Balarajah S et al. Axonally derived neuregulin-1 is required for remyelination and regeneration after nerve injury in adulthood. J. Neurosci. 31(9), 3225-3233 (2011).

- Demonstration of the role of NRG1 in remyelination and regeneration following peripheral nerve injury.

89. Chen Y, Hancock ML, Role LW, Talmage DA. Intramembranous valine linked to schizophrenia is required for neuregulin 1 regulation of the morphological development of cortical neurons. J. Neurosci. 30(27), 9199-9208 (2010).

90. Fricker Fr BJ, Zhu N, Garratt AN, Birchmeier C, Bennett DLH Neuregulin-1 is required for axoglial signalling following peripheral nerve injury to ensure normal re-myelination and functional recovery Presented at: The UK Neuromuscular Translational Research Conference, 2011. London, UK, 29-30 March 2011.

91. Farah MH, Pan BH, Hoffman PN et al. Reduced BACE1 activity enhances clearance of myelin debris and regeneration of axons in the injured peripheral nervous system. J. Neurosci. 31(15), 5744-5754 (2011).
92. Chen LE, Liu K, Seaber AV, Katragadda S, Kirk C, Urbaniak JR. Recombinant human glial growth factor 2 (rhGGF2) improves functional recovery of crushed peripheral nerve (a double-blind study). Neurochem. Int. 33(4), 341-351 (1998).

93. Yildiz M, Karlidag T, Yalcin S et al. Efficacy of glial growth factor and nerve growth factor on the recovery of traumatic facial paralysis. Eur. Arch. Otorhinolaryngol. 268(8), 1127-1133 (2011).

94. Joung I, Yoo M, Woo JH, Chang CY, Heo H, Kwon YK. Secretion of EGF-like domain of heregulin $\beta$ promotes axonal growth and functional recovery of injured sciatic nerve. Mol. Cell 30(5), 477-484 (2010).

95. Gao R, Zhang J, Cheng L et al. A Phase II, randomized, double-blind, multicenter, based on standard therapy, placebo-controlled study of the efficacy and safety of recombinant human neuregulin-1 in patients with chronic heart failure. J. Am. Coll. Cardiol. 55(18), 1907-1914 (2010).

96. Mahanthappa NK, Anton ES, Matthew WD. Glial growth factor 2, a soluble neuregulin, directly increases Schwann cell motility and indirectly promotes neurite outgrowth. J. Neurosci. 16(15), 4673-4683 (1996).

97. Li H, Wigley C, Hall SM. Chronically denervated rat Schwann cells respond to GGF in vitro. Glia 24(3), 290-303 (1998).

98. Calvo M, Zhu N, Tsantoulas C et al. Neuregulin-erbB signaling promotes microglial proliferation and chemotaxis contributing to microgliosis and pain after peripheral nerve injury. J. Neurosci. 30(15), 5437-5450 (2010).

99. Trachtenberg JT, Thompson WJ. Schwann cell apoptosis at developing neuromuscular junctions is regulated by glial growth factor. Nature 379(6561), 174-177 (1996).
100. Kopp DM, Trachtenberg JT, Thompson WJ Glial growth factor rescues Schwann cells of mechanoreceptors from denervation-induced apoptosis. J. Neurosci. 17(17), 6697-6706 (1997).

101. Grinspan JB, Marchionni MA, Reeves M, Coulaloglou M, Scherer SS. Axonal interactions regulate Schwann cell apoptosis in developing peripheral nerve: neuregulin receptors and the role of neuregulins. J. Neurosci. 16(19), 6107-6118 (1996).

102. Ebenezer GJ, McArthur JC, Thomas D et al. Denervation of skin in neuropathies: the sequence of axonal and Schwann cell changes in skin biopsies. Brain 130(10), 2703-2714 (2007).

103. Minwegen P, Friede RL. A correlative study of internode proportions and sensitivity to procaine in regenerated frog sciatic nerves. Exp. Neurol. 87(1), 147-164 (1985).

104. Fawcett JW, Keynes RJ. Peripheral nerve regeneration. Annu. Rev. Neurosci. 13, 43-60 (1990).

105. Huijbregts RP, Roth KA, Schmidt RE, Carroll SL. Hypertrophic neuropathies and malignant peripheral nerve sheath tumors in transgenic mice overexpressing glial growth factor $\beta 3$ in myelinating Schwann cells. J. Neurosci. 23(19), 7269-7280 (2003).

106. Zanazzi G, Einheber S, Westreich R et al. Glial growth factor/neuregulin inhibits Schwann cell myelination and induces demyelination. J. Cell. Biol. 152(6), 1289-1299 (2001).

107. Tapinos N, Ohnishi M, Rambukkana A. erbB2 receptor tyrosine kinase signaling mediates early demyelination induced by leprosy bacilli. Nat. Med. 12(8), 961-966 (2006).

- Important demonstration that leprosy bacilli directly bind to erbB2 receptors, causing strong activation and demyelination. 